\title{
Optimized Combined System of Shunt Active Power Filters and Capacitor Banks
}

\author{
Tri Desmana Rachmildha
}

\author{
School of Electrical Engineering and Informatics, Institute of Technology Bandung \\ Ganesha 10 Bandung, Indonesia \\ trides@konversi.ee.itb.ac.id
}

\begin{abstract}
In larges electrical system used in industries, office builidings, or hotels, capacitor banks are usually utilized to compensate the required VAR absorbed by the loads. As the nonliniear loads are increasing rapidly, the risk of resonance phenomenon occured is becoming higher as its terminal voltages are not anymore sinusoidal.

On the other hand, many customers have become aware to the problems caused by the harmonics due to the nonlinear loads. In this case, the active power filters (APFs) offer many interesting properties i.e. the elimination of harmonics, phase balancing feature and the power factor correction. The power factor correction feature suggests that the APFs are also able to compensate the VAR which means that the APFs are able to replace completely the capacitor banks. However, the higher reactive power occured in APFs is responsible to the higher current flowing in the switches, thus the higher rating of switches are required which leads to more expensive system.

In this paper, an optimized combined system between APF and capacitor bank is proposed. It is not necessary to dump the installed capacitor bank. In contrary, the capacitor bank will help the APF to compensate the VAR as the APF will concentrate mostly to eliminate the harmonics.
\end{abstract}

Keywords: instantaneous power theory, capacitor banks, active power filters.

\section{Introduction}

The increasing nonlinear loads connected to power system are causing many problems such as harmonic current problems. The injection of harmonic current will cause waveform distortion of system voltages, transformers heating, torque pulsation and heating in rotating machines, resonance phenomenon occured by the existance of capacitor bank, and so on. The resonance problem may happen in the power system where the capacitor bank is installed and the impact will be severe enough and sometimes lead to the system trip.

One of the solution of the harmonic problems can be solved using the passive filters consisting a tuned capacitance and inductance placed in series or parallel to the load. However, in this solution, certain harmonic will require its own passive filter, thus more harmonics to eliminate means more passive filters are required. Usually, only significant harmonics with lower frequencies will be eliminated, therefore the filters will be bulky due to inductor geometric size and imperfection in harmonic elimination due to the existance of higher harmonics.

Since the basic operation established in the 1970s, the active filters based on load currents for harmonic filtering have attracted many researchers and engineers who have concerned with harmonic pollution in power systems. The active filters inject the current harmonics needed by the loads so that the source will only deliver the fundamental current components. However, this technique cannot solve the imbalance problem and non unity power factor because the fundamental current components in each phase may have different magnitudes and may not be in phase with their corresponding voltages.

Received: April $25^{\text {th }}, 2011$. Accepted: October $2^{\text {nd }}, 2011$ 
As far as the author concern, the utilization of active filters comes with many topologies such as stand alone active filters, hybrid active and passive filters, and shunt or serial active filters. In the combined passive filters, the passive filters will be tuned to compensate significant harmonics with lower frequencies and the active filter itself is responsible for the higher harmonics. This technique may achieve lower current rating switches for the active filters.

In this paper, a similar principal used in combined passive and active filters is proposed. But different to it, a combined system of capacitor bank and shunt active power filter is proposed. Capacitor banks are usually already installed in large power systems such as in indsutries, hotels, or office building for VAR compensation. The used active filters are from active power filter (APF) kind which uses instantaneous power theory as its basic principe of operation. The APFs have many advantages compared to the conventional active filters such as harmonic elimination, phase balancing, and power factor correction. The objective of this technique is to avoid new installation of bulky passive filters, the utilization of existing capacitor bank, and to have better power quality in the system as offered by the APF's feature with reduced current rating in the APF itself.

There are two methods proposed in this paper to reduce the APF's current rating, one is based on constant VAR compensation and the other is based on minimization of fundamental current component.

\section{Compensation Strategy in Apf Using Instantaneous PQ0 Power Theory}

Power definition in the PQ0 instantaneous power theory [1-2] can be written as:

$$
\begin{aligned}
& {\left[\begin{array}{l}
p_{1} \\
q
\end{array}\right]=\left[\begin{array}{cc}
v_{\alpha} & v_{\beta} \\
-v_{\beta} & v_{\alpha}
\end{array}\right]\left[\begin{array}{l}
i_{\alpha} \\
i_{\beta}
\end{array}\right]} \\
& p_{0}=v_{0} i_{0}
\end{aligned}
$$

where all the values in $(\alpha \beta 0)$ reference can be obtained using the following transformation:

$$
\left[\begin{array}{l}
X_{\alpha} \\
X_{\beta}
\end{array}\right]=T 1 \cdot\left[\begin{array}{l}
X_{a} \\
X_{b} \\
X_{c}
\end{array}\right]
$$

with :

$$
\boldsymbol{T 1}=\sqrt{\frac{2}{3}}\left[\begin{array}{ccc}
1 & -1 / 2 & -1 / 2 \\
0 & \sqrt{3} / 2 & -\sqrt{3} / 2
\end{array}\right]
$$

and

$$
X_{0}=\sqrt{\frac{1}{3}}\left(X_{a}+X_{b}+X_{c}\right)
$$

where :

- $X_{a}, X_{b}, X_{c}$ : the voltage or current variables of the source in the (abc) reference,

- $X_{\alpha}, X_{\beta}, X_{0}$ : the voltage or current variables in ( $\left.\alpha \beta 0\right)$ reference.

- $p$ : instantaneous active power

- $q$ : instantaneous reactive power

- $p_{0}:$ zero sequence active power

The active power filter is based on the calculation of the powers absorbed by the load. Figure 1 shows the power compensation strategy used in APF in system with nonlinear load. 
The instantaneous active power is composed of average components [4,5], which will be permanently transferred from the source to the load. This components is represented by a unidirectional arrow in Figure 1. Another component is ripple component of instantaneous active power, and this component will be delivered by the APF to the load. Thus instantaneous active power can be expressed as:

$$
p=\bar{p}+\tilde{p}
$$

where the bar signs represent the average component and the tilde signs represent ripple component of instantaneous active power.

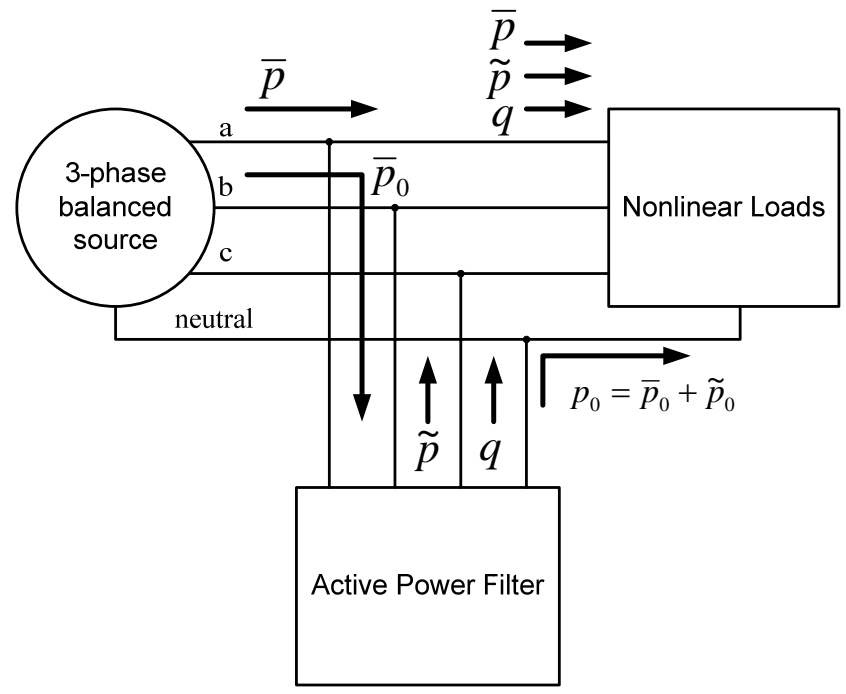

Figure 1. Power flow diagram

The component $p_{0}$ will only occur in a system with an unbalanced source. This $p_{0}$ will also have the average component, $\overline{p_{0}}$, and ripple components, $\widetilde{p_{0}}$. If $p_{0}$ exists, the compensation strategy is to deliver permanently its average component from the source to the APF first through the phase line and and the ripple component will be provided by the APF, as shown in Figure 1.

The same way, instantaneous reactive power also composed from average and ripple component, and can be expressed as:

$$
q=\bar{q}+\tilde{q}
$$

Based on Figure 1, the compensation done by active power filter can be implemented if the power components $\left(\overline{p_{0}},-\tilde{p},-q\right)$ are flowing in its phase lines and these power variables will become the reference values for the APF. The delivery of total $p_{0}$ from the APF to the load will be done by compensating the neutral line current. Thus the currents which should be flowing in the APF 3-phase lines can be denoted as:

$$
\left[\begin{array}{l}
i_{\alpha_{-} r e f} \\
i_{\beta_{-} r e f}
\end{array}\right]=\frac{1}{\left(v_{\alpha}^{2}+v_{\beta}^{2}\right)}\left[\begin{array}{cc}
v_{\alpha} & -v_{\beta} \\
v_{\beta} & v_{\alpha}
\end{array}\right]\left[\begin{array}{c}
p_{0}-\tilde{p} \\
-q
\end{array}\right]
$$

It can be shown from Figure 1 that, in the source side, there will be only constant active power thus the phase currents in the will be sinusoid, balanced, and in-phase with their corresponding voltages. 


\section{Combined system shunt APF and capacitor bank: constant var compensation [5]}

According to Eq. (8), the instantaneous reactive power $q$ will be provided by the APF thus it will increase the current

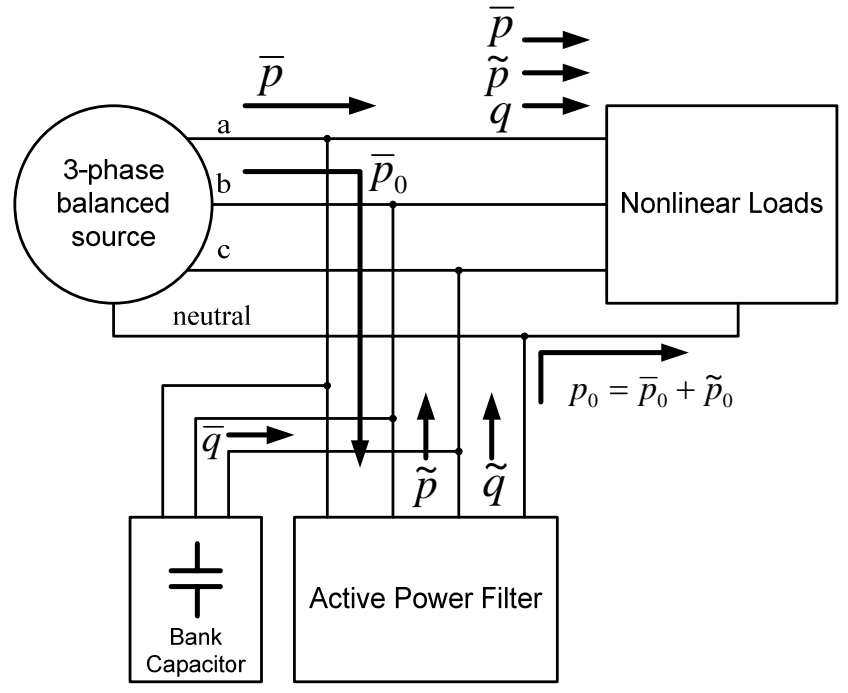

Figure 2. Power flow diagram using APF combined with capacitor bank, constan VAR compensation method

magnitude in APF. As shown in the Eqn. (7), it is possible to separate the average value of reactive power and its compensation will be done by the capacitor bank. Figure 2 shows the simple modification in power flow diagram.

To verify the proposed control method, digital simulation using PSIM has been carried out. Simulation scheme is shown in Figure 2. Switching frequency of $5 \mathrm{kHz}$ is chosen, while filter inductor $\left(\mathrm{L}_{\mathrm{fa}, \mathrm{b}, \mathrm{c}}\right)$ is $5 \mathrm{mH}$, DC capacitor in the converter is $2200 \mathrm{uf}$, line-to-line voltage is $380 \mathrm{~V}$.

PI current regulator is used for each leg of the APF.

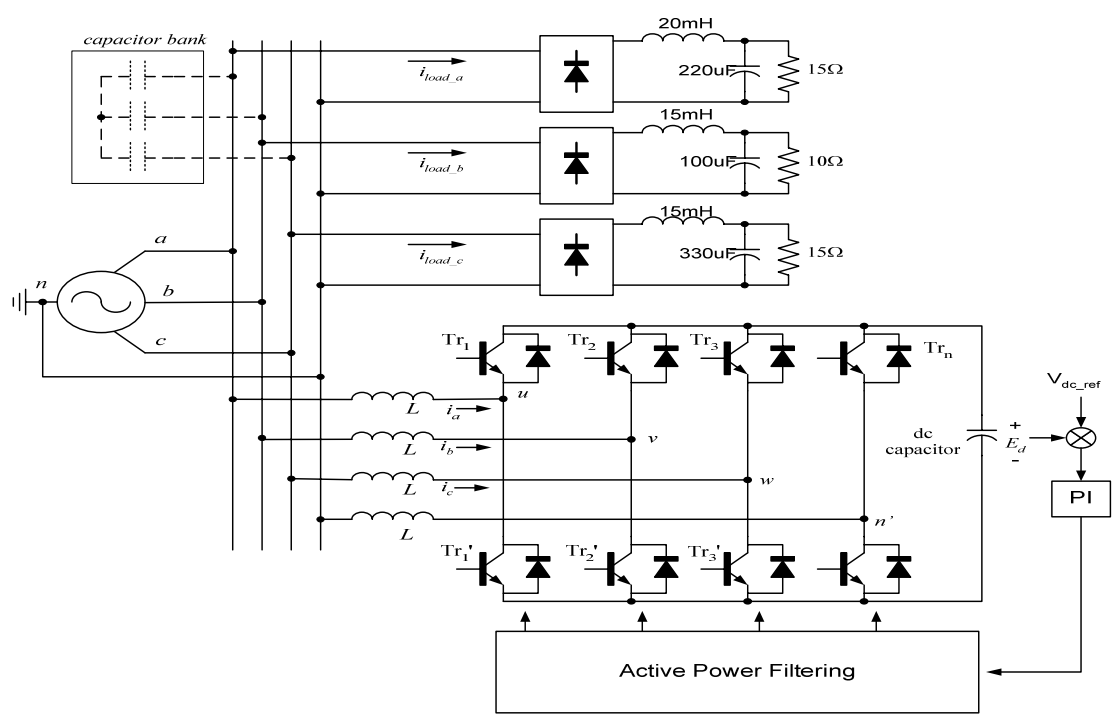

Figure 3. Simulation circuit 
Nonlinear and unbalanced loads are constructed using three single-phase rectifiers. Each single phase rectifier has different loads, as described in Figure 3.

The simulation is done firstly by compensating all the reactive power and the ripple part of the active power by the active power filter (without capacitor bank). The active power filtering is done pretty well as indicated by the source current waveforms compared to the load current, shown in Figure 4. The successful compensation for the neutral current is also shown in Figure 5.

Figure 6 shows the comparison between instantaneous active and reactive power absorbed by the load, and those delivered by the source. This figure shows that the APF is able to force the source to deliver only constant active power and zero reactive power, thus - because of the source is balanced - the source currents will be sinusoid and balanced with unity power factor. Figure 7 shows the current waveforms which are delivered by the APF, and for phase a, b, and c, their rms values are $9.95 \mathrm{~A}, 7.41 \mathrm{~A}$, and 11.03 A respectively.
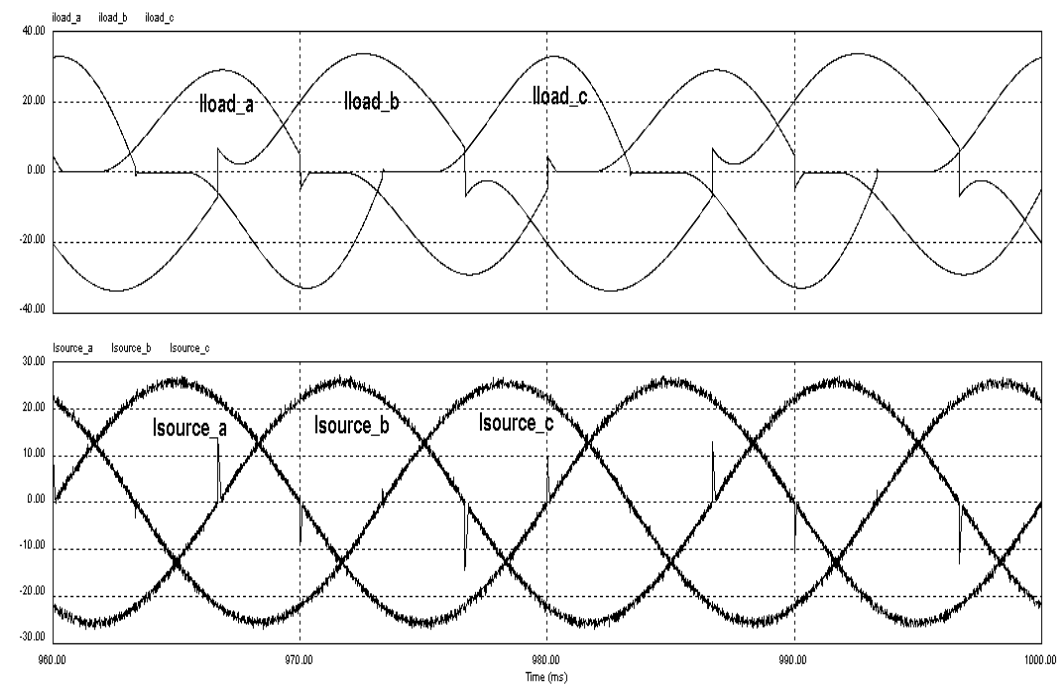

Figure 4. Load current (above) and source current (below) waveforms; full compensation by the active power filter.

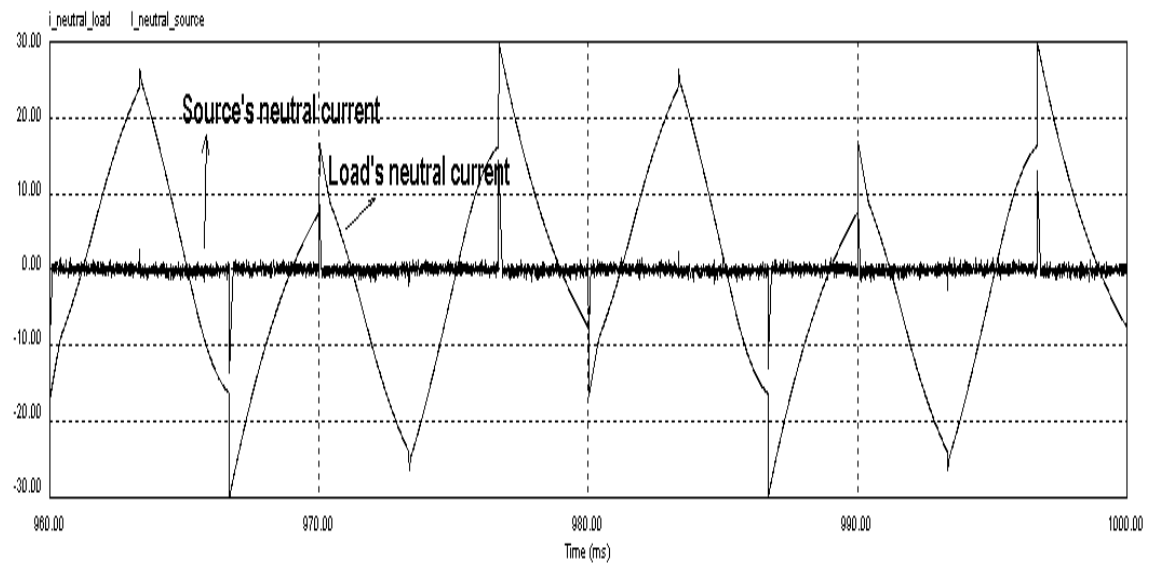

Figure 5. Neutral current in the source side and load side 


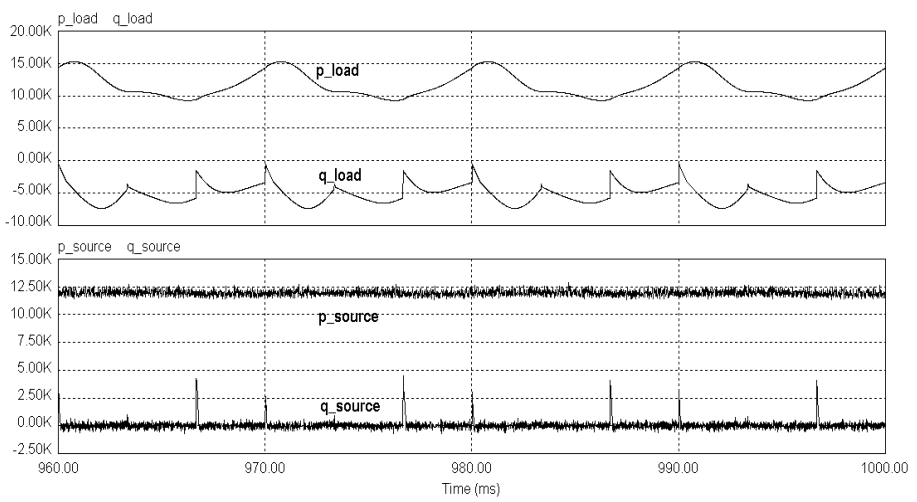

Figure 6. Instantaneous active and reactive power of the load side (above) and the source side (below); full compensation by the active power filter.

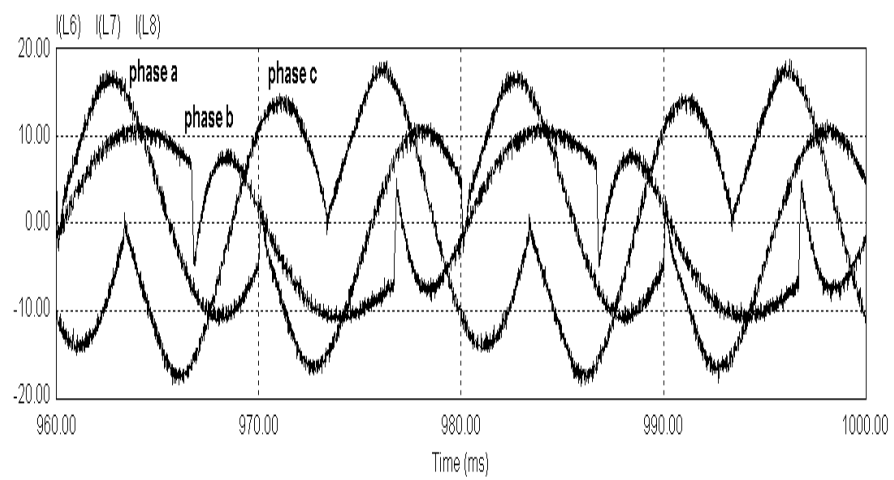

Figure 7. Waveforms of the currents delivered by the active power filter.

The second simulation is done using 3 identical capacitors as a representation of a capacitor bank, connected at the load side as shown in Figure 3. The value of each capacitor is calculated based on the dc component of the reactive power absorbed by the load. In this example, the dc value of the reactive power is 5105 VAR. The required capacitance can be calculated as:

$$
C=\frac{3 V^{2}}{\omega Q_{\text {avg }}}
$$

where :

$C:$ is the required capacitance in each phase line.

$V:$ is the line to neutral voltage in rms value

$Q_{a v g}:$ is the average component of $\mathrm{q}$

From Eq. (9), each capacitor will be valued about $112 \mu \mathrm{F}$. These capacitors will compensate the constant reactive power needed by the load, while the APF will compensate only the ripple component.

Figure 8 shows that the active filtering is done successfully, as the source has sinusoid current waveforms and delivers constant active power and zero reactive power.

The compensating currents transmitted from the APF are shown in Figure 9. The rms values of these currents are 5.52 A, 5.02, and 6.26 A for phase a, b, and c respectively. Thus, compared to the previous system, the rating current for the APF can be reduced significantly. 

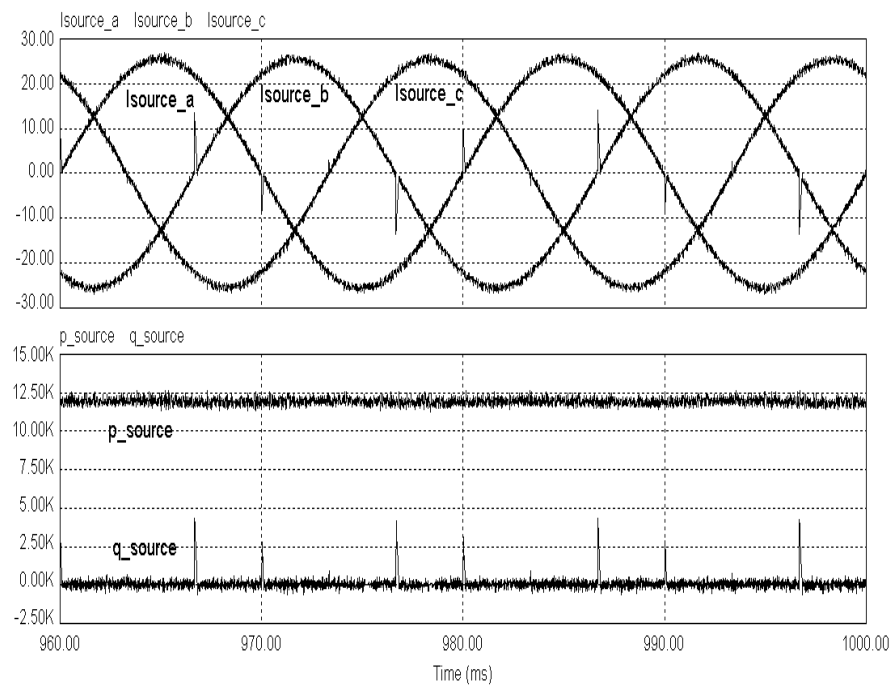

Figure 8. Source current waveforms (upper) and instantaneous active and reactive power (below) using combined APF and capacitor bank

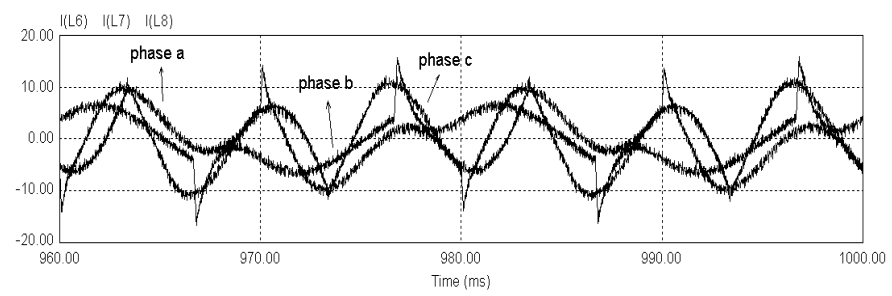

Figure 9. Current waveforms of the APF

\section{Combined system shunt APF and capacitor bank: minimization of fundamental} current

This second method is based on the reduction of fundamental component of current in APF. According to Eq. (8), it is possible to have the current which should flow in each phase of the APF, as:

$$
\left[\begin{array}{l}
i_{a f} \\
i_{b f} \\
i_{c f}
\end{array}\right]=\mathrm{T}_{1}^{-1}\left[\begin{array}{l}
i_{\alpha_{\alpha} r e f} \\
i_{\beta_{-} r e f}
\end{array}\right]
$$

Where $i_{a f}, i_{b f}$, and $i_{c f}$ are reference currents for the APF in (abc) reference system.

The proposed method deals with extracting the fundamental components of each APF phase current using the FFT and then replacing it by the simple phasor summation between current injected by a capacitor and the rest will be provided again by the APF.

Since the capacitor will inject current exactly $90^{\circ}$ leading to their corresponding voltages, it is possible to draw capacitor current reference frame. Figure 10 shows the capacitor current reference frame (dotted line arrows) and an example of fundamental component of currents which should be provided by the APF (solid line arrow). It is possible to do the projection of these currents to the capacitor current reference frame. 


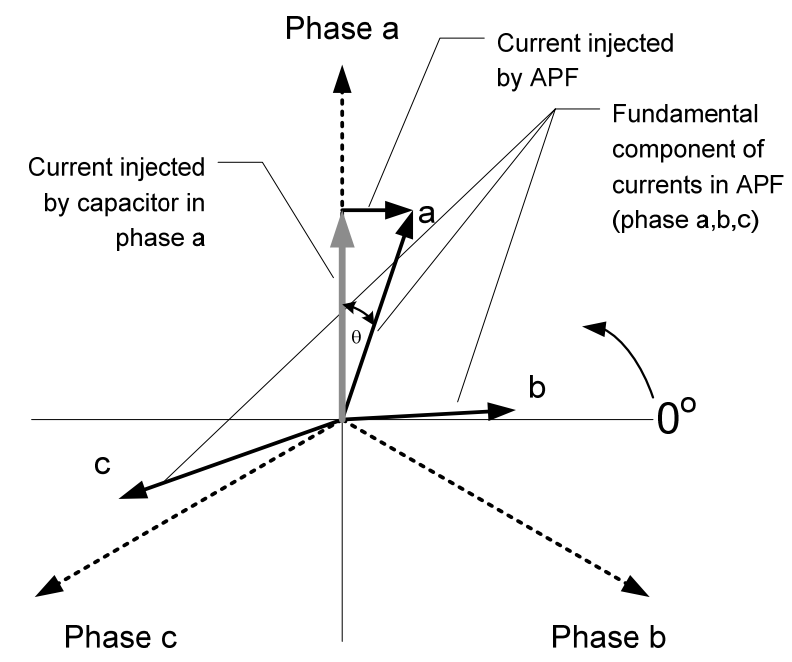

Figure 10. Capacitor current reference and an example of fundamental component of currents which should be injected by the APF

In Figure 10, the fundamental component of APF currents can be decomposed into 2 phasors as done in phase a, the first one is the projection onto corresponding phase, and the second phasor is orthogonal to the first one. This first one will be the current provided by the capacitor and the second one will be provided by the APF. It is shown in Figure 10 that the current provided by APF will be smaller than the original one.

Using this technique, the capacitor current and its ortoghonal component will be:

$$
\begin{aligned}
& i_{C}=i_{A P F} \cos (\theta) \\
& i_{A P F \_n e w}=i_{A P F} \sin (\theta)
\end{aligned}
$$

Where:

- $i_{C}$ : the capacitor current

- $i_{A P F}$ : fundamental component of APF's current which shoul be provided

- $i_{A P F}$ new: fundamental component which will be provided by APF after working together with the capacitor

- $\theta$ : the angle between corresponding capacitor current reference and iAPF.

Using the same example as the previous simulation, it is possible to calculate the capacitor currents $i_{C}$ for each phase and the new APF currents, $i_{A P F_{-} \text {new }}$, as shown in Table 1.

Table 1. An example of APF's new fundamental current calculation

\begin{tabular}{|c|r|r|r|r|r|r|r|}
\hline \multirow{2}{*}{ Phase } & \multicolumn{2}{|c|}{ APF's fundamental } & \multirow{2}{*}{$\theta$} & \multicolumn{2}{c|}{ Capacitor Current } & \multicolumn{2}{c|}{ APF's new fund. } \\
\cline { 2 - 6 } \cline { 5 - 8 } & mag & angle & & mag & angle & APF & angle \\
\hline a & 12.47 & 71.35 & 19 & 11.81585 & 90 & 3.985785 & 0 \\
\hline b & 9.8 & 3.15 & -33 & 8.206541 & -30 & -5.35655 & -120 \\
\hline c & 13.37 & -160.5 & 371 & 13.15403 & 210 & 2.39338 & 120 \\
\hline
\end{tabular}

The new fundamental component for APF's current should be injected and the obtained capacitor current can be used to calculate the capacitor which should be installed in each phase, using the following equation. 


$$
C=\frac{I_{C \_} m a g}{\sqrt{2} \omega V}
$$

where:

$C$ : the capacitance of capacitor which should be installed in corresponding phase $I_{C_{-} \text {mag }}$ : maximum value of obtained capacitor current

$V$ : line to neutral voltage (where the capacitor will be installed).

In this example, the capacitors will be:

- $120.45 \mu \mathrm{F}$ in phase a

- $83.66 \mu \mathrm{F}$ in phase b

- $134.10 \mu \mathrm{F}$ in phase c

Note that in each phase, the capacitors will be different.

The result of the simulation using this technique can be shown in Figure 11.

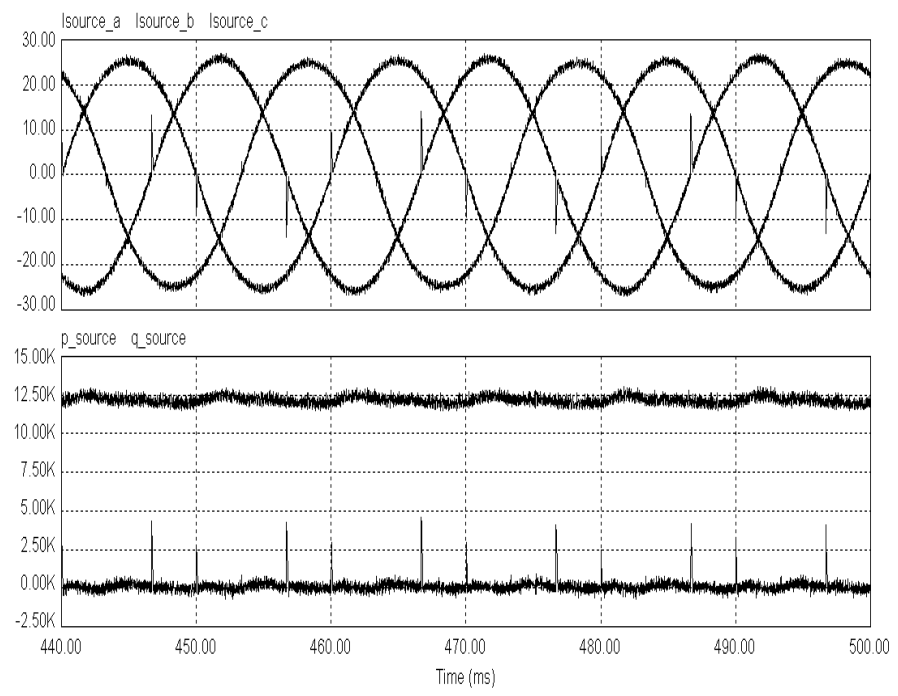

Figure 11. Source current waveforms (upper) and the instantaneous active and reactive power plot

Figure 11 indicates that the technique can successfully implemented where the source current waveforms are sinusoidal and balanced the active power is relatively constant and the reactive power is practically equals to zero.

The APF's phase currents can be shown in Figure 12.

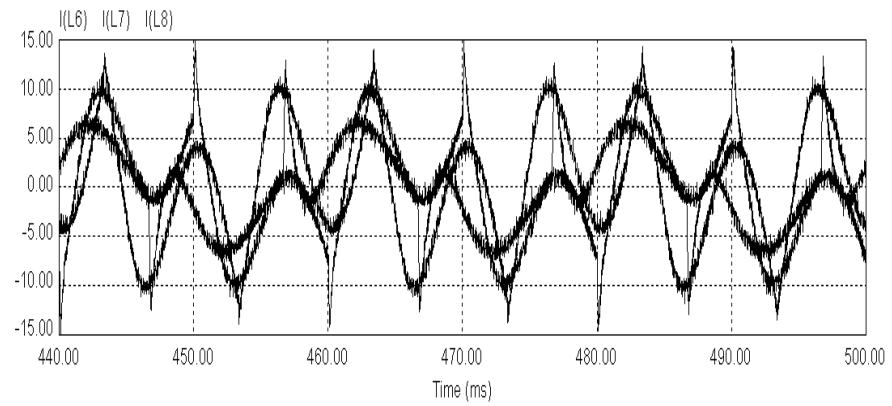

Figure 12. APF's phase currents 
The APF phase currents are measured $5.67 \mathrm{~A}, 4.33 \mathrm{~A}$, and $6.14 \mathrm{~A}$ for phase a,b,c respectively. Compared to the constant VAR compensation method, the average phase current is reduced to about $4 \%$ if this technique is used.

\section{Conclusion}

A study of combined system between existing capacitor bank and active power filter has been proposed. Combination between capacitor and active power filter has successfully maintained the active power filtering concept while other advantage can be achieved. Using this method will lead to smaller current capacity carried by the active power filter. This will decrease overall size and cost of the active power filter because of the utilization of smaller rating component and lower loss. The other benefit of this combined system is the elimination of harmonic current flowing in the source side thus decreasing the possibility of harmonic resonance occurred in the system especially a system with capacitor bank.

\section{References}

[1] M.C. Benhabib, S. Saadate, New control approach for four-wire active power filter based on the use of synchronous reference frame, www.sciencedirect.com, 2004

[2] H. Akagi, S. Ogasawara, H. Kim, The Theory of Instantaneous Power in Three Phase Four-Wire Systems : A Comprehensive Approach

[3] F.Z. Peng, J. Lai, Generalized Reactive Power Theory for Three-Phase Power Systems, IEEE Transactions on Instrumentation and Measurement, Vol. 45, No. 1, February 1996

[4] J. Afonso, C. Couto, J. Martins, Active Filters with Control Based on the p-q Theory, IEEE Industrial Electronics Society Newsletter vol. 47, no. 3, Sept 2000.

[5] H. Kim, F. Blaabjerg, B. Bak-Jensen, J. Choi, Instantaneous Power Compensation in Three-Phase systems by Using p-q-r Theory.

[6] Tri D. Rachmildha, A. Rizqiawan, Y. Haroen, “A Study of Combined System of Capacitor Bank and Shunt Active Power Filter”, ICEEI International Conference, UKM Malaysia, 2009.

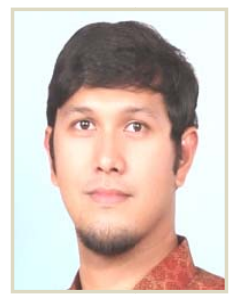

Tri Desmana Rachmildha, Lecturer at STEI-ITB, Graduate, Electrical Engineering option Electronics, 1993-1998, Bandung Institute of Technology. Master degree, Electrical Engineering, option Power Engineering, 1999 - 2002, Bandung Institute of Technology. Doctorate degree, 2009, Joint PhD Supervision Program between Insitut Nationale Polytechnique de Toulouse - Ecole Nationale Superieure d'Electrotechnique, d'Electronique, d'Informatique, d'Hydrolique, et de Telecommunication (INPT -ENSEEIHT, France) and School of Electrical Engineering and Informatics, Bandung Institute of Technology, Indonesia. He has papers Published in International and National Publication. 